\title{
Manifestações orais associadas à infecção por COVID-19
}

\author{
Oral manifestations associated with COVID-19 infection \\ Manifestaciones orales asociadas con infección por COVID-19
}

Recebido: 30/11/2021 | Revisado: 04/12/2021 | Aceito: 10/12/2021 | Publicado: 18/12/2021

Maria Itala Otacilia Silva Barros ORCID: https://orcid.org/0000-0002-7259-7796 Centro Universitário Cesmac, Brasil E-mail: italabarros2@gmail.com

Guilherme Levy Omena Firmino ORCID: https://orcid.org/0000-0003-3641-0864 Centro Universitário Cesmac, Brasil E-mail: guilhermeomena@gmail.com Thaís da Silva Vieira ORCID: https://orcid.org/0000-0001-5022-7738 Centro Universitário Cesmac, Brasil E-mail: thais.kiyo@gmail.com

Andreza de Albuquerque Araujo ORCID: https://orcid.org/0000-0001-7149-6558 Centro Universitário Cesmac, Brasil

E-mail: albuquerqueandreza200@gmail.com Laís de Lima Barros Souza ORCID: https://orcid.org/0000-0002-5881-5769 Centro Universitário Cesmac, Brasil E-mail: laisbarros132@gmail.com

Beatriz Santos Reis

ORCID: https://orcid.org/0000-0002-9407-2114 Centro Universitário Cesmac, Brasil E-mail: beatriz70reis@gmail.com Karoline Gouvêa de Souza

ORCID: https://orcid.org/0000-0002-2496-5558 Centro Universitário Cesmac, Brasil E-mail: karollgouveas@gmail.com

Catarina Rodrigues Rosa de Oliveira ORCID: https://orcid.org/0000-0001-9178-8902 Centro Universitário Cesmac, Brasil

E-mail: catarinarosaodonto@hotmail.com

\begin{abstract}
Resumo
O objetivo desta revisão de literatura foi verificar a relação do surgimento de coinfecções bucais decorrentes ou junto à infecção por COVID-19 em pacientes. Detectado pela primeira vez no final de 2019 e rapidamente se tornou um surto global, sendo denominado de SARS-CoV-2, o vírus é transmitido de um indivíduo para o outro por gotículas de saliva, os sintomas se apresentam como perda de paladar, olfato, febre, tosse e falta de ar. Novas descobertas acerca do vírus surgem diariamente e apesar de controversas na literatura, diversas alterações e lesões orais foram descritas como possíveis manifestações orais de COVID-19, como disgeusia, úlceras orais, petéquias, máculas avermelhadas, gengivite descamativa, entre outros. Além disso, a doença também foi citada como a causa de alterações sistêmicas, comprometimento do sistema imune e predisponentes a coinfecções oportunistas, podendo ocorrer como resultado direto ou indireto pOR infecção do SARS-CoV-2. Contudo, verificou-se que mais estudos são necessários para orientar e esclarecer possíveis alterações bucais e o estabelecimento de causa e efeito.
\end{abstract}

Palavras-chave: Manifestações bucais; COVID-19; SARS-CoV-2; Mucosa bucal; Medicina bucal.

\begin{abstract}
The aim of this descriptive study was to verify the relationship between the appearance of oral coinfections resulting from or together with COVID-19 infection in patients. First detected in late 2019 and quickly became one of the global ones, being called SARS-CoV-2, the virus is transmitted from one individual to another by droplets of saliva, the symptoms present themselves as loss of taste, smell, fever, coughing and shortness of breath. New discoveries about the virus arise daily and despite controversies in literature, several changes in oral health and injuries were obtained as oral manifestations of COVID-19, such as dysgeusia, oral ulcers, petechiae, reddened macules, desquamative gingivitis, others. In addition, the disease was also cited as a cause of systemic changes, compromised immune system and predisposing factors to opportunistic coinfections, which may occur as a direct or indirect result
\end{abstract}


of SARS-CoV-2 infection. However, it was found that more studies are needed to guide and clarify possible changes in oral health and the property of cause and effect.

Keywords: Oral manifestations; COVID-19; SARS-CoV-2; Mouth mucosa; Oral medicine.

\section{Resumen}

El objetivo de esta revisión de la literatura fue verificar la relación entre la aparición de coinfecciones orales como resultado o junto con la infección por COVID-19 en pacientes. Detectado por primera vez a fines de 2019 y rápidamente se convirtió en un brote global, siendo llamado SARS-CoV-2, el virus se transmite de un individuo a otro por gotitas de saliva, síntomas presentes como pérdida del gusto, olfato, fiebre, tos y dificultad para respirar. Diariamente surgen nuevos descubrimientos sobre el virus y, a pesar de ser controvertidos en la literatura, se han descrito varias alteraciones y lesiones orales como posibles manifestaciones orales del COVID-19, como disgeusia, úlceras orales, petequias, máculas enrojecidas, gingivitis descamativa, entre otras. Además, la enfermedad también fue citada como la causa de cambios sistémicos, sistema inmunológico comprometido y factores predisponentes a coinfecciones oportunistas, que pueden ocurrir como resultado directo o indirecto de la infección por SARS-CoV-2. Sin embargo, se encontró que se necesitan más estudios para orientar y aclarar posibles cambios orales y el establecimiento de causa y efecto.

Palabras clave: Manifestaciones bucales; COVID-19; SARS-CoV-2; Mucosa bucal; Medicina oral.

\section{Introdução}

Um novo coronavírus foi detectado pela primeira vez no final de 2019 e rapidamente se tornou um surto global, sendo denominado de SARS-CoV-2 (Liu \& Shih, 2020) e posteriormente declarado como pandemia pela Organização Mundial da Saúde (OMS) desde 11 de março de 2020.

No mundo, foram confirmados 261.075.046 casos da doença e um total de 5.195.138 óbitos sendo 22.076.863 casos confirmados apenas no Brasil, e um total de 614.186 óbitos. Dados esses obtidos até 28 de novembro de 2021.

A COVID-19 é transmitida de pessoa para pessoa, por gotículas de saliva, espirro, tosse, assim como pelo contato da boca, nariz ou olhos, ou até mesmo, por meio de objetos e superfícies contaminadas. Embora um quadro clínico grave seja mais provável em indivíduos com comorbidades, pessoas de todas as idades são susceptíveis à doença (Chen et al., 2020). A sintomatologia desses pacientes, incluindo febre, mal-estar, tosse seca e dispneia foram diagnosticados como pneumonia viral (Huang et al., 2020) Considerados comuns os distúrbios gustatórios e olfativos (Passarelli et al., 2020).

O SARS-CoV-2 tem se mostrado um vírus neurotrópico e mucotrópico, podendo afetar o funcionamento das glândulas salivares, as sensações de paladar, olfato e a integridade da mucosa oral (Lechein et al., 2020). Estudos indicariam que esse novo coronavírus tem a capacidade de alterar o equilíbrio da microbiota oral, o que, combinado com um sistema imunológico deprimido, permitiria a colonização por infecções oportunistas (Dziedzic \& Wojtyczka, 2020).

Diferentes doenças virais podem acarretar problemas ao sistema estomatognático, diretamente ou de maneira secundária; as manifestações podem variar, mas, no geral, aparecem como ulcerações ou bolhas nos tecidos bucais (Santosh \& Muddana, 2020).

Também não é possível afirmar que os achados bucais em pacientes infectados com SARS-CoV-2, relatos na literatura, se tratem de manifestações diretas da COVID-19. A publicação de material que fale a respeito da ocorrência de alterações bucais da COVID-19 ainda é muito recente na literatura e, assim, pode variar de maneira significativa. Além do mais, as doenças de base, tanto sistêmicas quanto locais, podem ser fator de contribuição para as lesões de cavidade oral (Pedrosa \& Nogueira, 2020).

O objetivo desse estudo é abordar a possível associação das manifestações orais com a infecção pelo vírus SARSCoV2 e quais as manifestações que podem surgir durante a infecção, tendo uma relevância social significativa para que possamos contribuir para novas hipóteses sobre esse tema tão presente na realidade atual. 


\section{Metodologia}

Trata-se de um estudo de revisão de literatura cuja pergunta do estudo foi elaborada utilizando-se a estratégia PICO (Santos et al., 2007) em que atribui-se ao P (população) pacientes com COVID-19 que apresentam manifestações orais, I (indicador) a avaliação estomatológica na busca por lesões em mucosa e gengiva, C (controle) pacientes com COVID-19 que não apresentam manifestações bucais e O (desfecho) relação entre as manifestações orais e prognóstico da saúde sistêmica e quais as alterações bucais mais frequentes em indivíduos com COVID-19. Assim sendo a pergunta do estudo é: “quais as relações entre a COVID-19 e as manifestações orais no que se diz respeito às áreas acometidas na cavidade bucal e o prognóstico sistêmico dos pacientes com a infecção?”.

A busca eletrônica de artigos foi realizada em agosto de 2021, seguindo o Guia PRISMA (Principais Itens para Relatar Revisões sistemáticas e Metaanálises) (Galvão et al., 2015) nas plataformas PubMed e BVS envolvendo estudos publicados a qualquer ano, sem restrições de idioma e país. Os descritores utilizados foram “Oral Manifestations”, "COVID19" e "'Mouth Mucosa', adquiridos do diretório dos Descritores em Ciências da Saúde (DeCS), usados em combinação com auxílio do operador booleano "AND".

Sendo selecionados 65 artigos, posteriormente foi feita uma filtragem e leitura desses artigos, resultando na escolha de 21 artigos, para ser composta a revisão de literatura.

Os critérios de inclusão dos artigos na pesquisa foram os relatos de revisões sistemáticas, séries de casos, carta ao editor e revisão narrativa compreendidos nos anos de 2020 e 2021.

Foram excluídas publicações depois da leitura do trabalho por não se adequarem ao objetivo do estudo, além dos que não estavam relacionados com o tema e os duplicados.

\section{Resultados e Discussão}

A cavidade bucal é considerada "portal de entrada", vendo que é o meio entre o corpo e o exterior. Sendo assim, local de risco de infecção e transmissão de microrganismos. Sabe-se também que o SARS-CoV2 tem receptores específicos com os quais se liga à cavidade oral.

Pelo menos metade dos pacientes apresenta quadros leves a moderados de alterações de paladar, sendo está uma das manifestações mais comumente relatadas, bem como lesões em mucosa oral. Estas manifestações podem inclusive acontecer antes mesmo das manifestações respiratórias da doença, podendo acontecer o seu surgimento entre 2-24 dias após o início do contato com o vírus. Sabe-se ainda que quanto maior a carga viral, maior é a gravidade da doença. Sendo assim, o controle da sintomatologia pode ajudar a diminuir os quadros mais graves da doença.

As lesões em mucosas bucais aparentam ocorrer como coinfecções, devido à baixa de imunidade do paciente afetado pelo novo coronavírus ou como efeito colateral do tratamento da doença, seja por conta das diversas medicações ou da necessidade de intubação do paciente mais grave. Por isso, as alterações de paladar, podendo em pacientes acometidos pelo coronavírus, o aparecimento de lesões em mucosa bucal, isoladamente, não é um indicativo de suspeita de COVID-19.

O tratamento para tais coinfecções são pré-existentes e o mesmo deve ser indicado individualmente pelo cirurgiãodentista, a depender de cada caso. Em relação às alterações de paladar, por ainda não se conhecer a etiopatogenia do quadro, não se tem tratamento específico, sendo observado o desaparecimento espontâneo do sintoma durante a recuperação do paciente.

As lesões erosivas ou ulcerativas se apresentam clinicamente como lesões dolorosas com bordas irregulares sendo geralmente encontradas ou observadas na língua, mucosa labial e palato (Santos et al., 2021; Iranmanesh et al., 2021; Sinadinos \& Shelswell, 2020; Brandão et al., 2020; Riad et al., 2020; Hocková et al., 2021). Santos et al., (2020) relatam que o 
surgimento das lesões ulcerativas ocorre devido a presença de patógenos oportunistas ou por meio de coinfecções; podendo ser as úlceras herpéticas, as úlceras necróticas hemorrágicas e úlceras de pressão perioral.

As lesões ulceradas surgem cinco dias após o agravamento dos sintomas respiratórios e foram tratadas com antivirais e o uso de laser para terapia de potência e bioestimulação tecidual (Brandão et al., 2020; Hocková et al., 2021). Estudos adicionais precisam ser feitos para investigar se a infecção pelo coronavírus é o fator causal para o surgimento das lesões ulceradas ou se aparecem devido a deficiência do sistema imune e as condições de higiene bucal do paciente (Brandão et al., 2020; Hocková et al., 2021).

Os artigos estudados mostraram que para o tratamento dessa infecção os pacientes utilizaram medicamentos como fluconazol sistêmico ou nistatina tópica de acordo com a gravidade e a superfície da lesão (Hocková et al., 2021). Vale enfatizar que infecções como a candidíase podem se tornar um risco de vida se agravadas (Hocková et al., 2021; Riad et al., 2020).

Na literatura analisada há poucos estudos que comprovem que a presença de lesões orais agrava o quadro sistêmico do paciente. No entanto, é importante conhecer as lesões, pois muitas delas precedem sintomas respiratórios típicos da COVID-19 além de que, o agravamento das lesões orais antecede um quadro clínico sistêmico mais sério (Brandão et al., 2020).

Contudo, Iranmanesh et al. (2021) mostra que as manifestações clínicas orais e os sintomas sistêmicos da COVID-19 apareceram de forma simultânea e que existe uma relação da gravidade da doença com a presença de lesões orais mais severas e disseminadas. A imunossupressão secundária a infecção por COVID-19 também pode ser um fator contribuinte para o surgimento de infecções orais oportunistas que dependendo da condição sistêmica do paciente tornam-se sinérgicas com quadros patológicos potencialmente fatais (Riad et al., 2020; Iranmanesh et al., 2021).

Recalcati (2020) descreveu que 20,4\% de 88 pacientes com COVID-19 avaliados por sua pesquisa apresentavam algum tipo de manifestação cutânea, como erupção cutânea eritematosa, urticária generalizada e vesículas. Casas et al. (2020) relataram ainda que alguns pacientes apresentavam outras manifestações, também na cavidade oral, como enantema.

Em um atlas publicado como material suplementar, os autores apresentaram o caso de um paciente com erupção maculopapular na mucosa oral localizada no palato, gengiva bucal inferior e mucosa do lábio inferior (Casas et al., 2020). Eles sugeriram que as lesões cutâneas eram semelhantes às encontradas em doenças virais comuns, mas enfatizaram que estudos adicionais são necessários para confirmar sua associação com COVID-19.

Em relação ao COVID-19, apenas dois relatórios tratam especificamente de enantema oral. Carreras-Presas et al. (2020) descreveram lesões orais em um paciente confirmado e dois suspeitos; o paciente confirmado apresentava bolhas na mucosa labial interna, gengivite descamativa e erupção cutânea generalizada, enquanto os outros dois casos apresentavam úlceras palatinas dolorosas semelhantes a lesões herpéticas. Enquanto Chaux-Bodard et al. (2020) relataram uma lesão oral como um possível sintoma inaugural de COVID-19 em um paciente que exibia inflamação dolorosa das papilas da língua que evoluiu para uma mácula eritematosa e, subsequentemente, para uma úlcera assintomática. Uma lesão eritematosa na pele se desenvolveu o teste diagnóstico positivo.

Mais estudos são necessários para entender a patogenia do vírus e as suas implicações sistêmicas, embora note-se que a maioria das pesquisas apresenta conclusões controversas sobre o surgimento das manifestações orais. No entanto, é necessário que o exame bucal dos pacientes com COVID-19 não seja negligenciado para maiores descobertas e entendimento acerca da doença, bem como a existência de um atendimento multidisciplinar para garantir um melhor prognóstico e uma maior qualidade de vida desses pacientes (Santos et al., 2021; Sinjari et al., 2020; Brandão et al., 2020). 


\section{Considerações Finais}

Baseado no estudo de vários artigos utilizados para produzir a revisão de literatura os resultados mostraram que úlceras orais e suas variações, a candidíase, xerostomia e a disgeusia são as manifestações orais mais presentes em pacientes com testes positivos para a COVID-19, acometendo principalmente os sítios da língua, mucosa labial e palato. Acredita-se que o surgimento das úlceras e da disgeusia seja devido à capacidade do vírus SARS-CoV-2 se ligar a enzima ACE2 e já o aparecimento da candidíase devido ao comprometimento do sistema imune.

Mais estudos clínicos e randomizados precisam ser feitos para ser possível estabelecer uma real relação de causa e efeito e associar o prognóstico do paciente a presença de manifestações orais, já que estas parecem indicadores importantes para fins diagnósticos e de acompanhamento clínico. Estudos em larga escala, realizados com pacientes diagnosticados com COVID-19, com comprometimento clínico diverso, e com intervenções odontológicas, comparados com resultados de pacientes que não tenham acesso à essa intervenção, podem contribuir com a obtenção de resultados baseados em evidências científicas robustas.

\section{Referências}

ANVISA. (2021). Nota Técnica GVIMS/GGTES/ANVISA No 07/2020. Orientações para prevenção e vigilância epidemiológica das infecções por SARSCoV-2 (COVID-19) dentro dos serviços de saúde. Ministério da Saúde. https://www.gov.br/anvisa/ptbr/centraisdeconteudo/publicacoes/servicosdesaude/notas-tecnicas/nota-tecnica-gvims-ggtes-anvisa-no-07-2020 .

Brandão, T., Gueiros, L., Melo, T., Prado-Ribeiro, A., Froelich, A., Nesrallah, A., Prado, G., Santos-Silva, A. \& Migliorati, C. (2021). Oral lesions in patients with SARS-CoV-2 infection: could the oral cavity be a target organ?. Oral Surgery, Oral Medicine, Oral Pathology And Oral Radiology, 131 (2),45-51. http://dx.doi.org/10.1016/j.oooo.2020.07.014.

Carreras-Presas, C., Sánchez, J., López-Sánchez, A., Jané-Salas, E. \& Pérez, M. (2020). Oral vesiculobullous lesions associated with SARS-CoV-2 infection. Oral Diseases, 27(3), 710-712. http://dx.doi.org/10.1111/odi.13382.

Casas, C., Catala, A., Hernández, G., Rodríguez-Jiménez, P., Fernández-Nieto, D., Lario, A., Fernández, I., Ruiz-Villaverde, R., Falkenhain-López, D., Velasco, M., García-Gavín, J., Baniandrés, O., González-Cruz, C., Morillas-Lahuerta, C., Cubiró,X., Nart, I, Selda-Enriquez, G., Romaní, J., Fustà-Novell, X., Melian-Olivera A., Riesco, M., Burgos-Blasco, P., Ortigosa, J., Rodriguez, M. \& García-Doval, I. (2020). Classification of the cutaneous manifestations of COVID -19: a rapid prospective nationwide consensus study in spain with 375 cases. British Journal Of Dermatology, 183(1), 71-77. http://dx.doi.org/10.1111/bjd.19163.

Chaux-Bodard, A., Deneuve, S. \& Desoutter, A. (2020). Oral manifestation of Covid-19 as an inaugural symptom? Journal Of Oral Medicine And Oral Surgery, 26(2), 18. http://dx.doi.org/10.1051/mbcb/2020011.

Chen, N., Zhou, M., Dong, X., Qu, J., Gong, J., Han, Y., Qiu, Y., Wang, J., Liu, Y., Wei, Y., Xia, J., Yu, T., Zhang, X. \& Zhang, L. (2020). Epidemiological and clinical characteristics of 99 cases of 2019 novel coronavirus pneumonia in Wuhan, China: a descriptive study. The Lancet, 395(10223), 507-513. http://dx.doi.org/10.1016/s0140-6736(20)30211-7.

Dziedzic, A \& Wojtyczka, R. (2020). The impact of coronavirus infectious disease 19 (COVID-19) on oral health. Oral Diseases, (27)3, 703-706. http://dx.doi.org/10.1111/odi.13359.

Hocková, Barbora, Riad, A., Valky, J., Šulajová, Z., Stebel, A., Slávik, R., Bečková, Z., Pokorná, A., Klugarová, J. \& Klugar, M. (2021). Oral Complications of ICU Patients with COVID-19: case-series and review of two hundred ten cases. Journal Of Clinical Medicine, 10(4), 581. http://dx.doi.org/10.3390/jcm10040581.

Huang, Chaolin, Wang, Y., Li, X., Ren, L., Zhao, J., Hu, Y., Zhang, L., Fan, G., Xu, Y., Gu, X., Cheng, Z., Yu, T., Xia, J., Wei, J., Wu, W, Xie, X., Yin, W., Li, H., Liu, M., Xiao, Y., Gao, H., Guo, L., Xie, J., Wang, G., Jiang, R., Gao, Z., Jin, Q. Wang, J. \& Cao, B. (2020). Clinical features of patients infected with 2019 novel coronavirus in Wuhan, China. The Lancet, 395(10223), 497-506. http://dx.doi.org/10.1016/s0140-6736(20)30183-5.

Iranmanesh, B., Khalili, M., Amiri, R., Zartab, H. \& Aflatoonian, M. (2020). Oral manifestations of COVID -19 disease: a review article. DermatologicTherapy, 34(1), 14578. http://dx.doi.org/10.1111/dth.14578.

Lechien, J., Chiesa-Estomba, C., Siati, D., Horoi, M., Bon, S., Rodriguez, A., Dequanter, D., Blecic, S., Afia, F., Distinguin, L.,Chekkoury-Idrissi, Y., Hans, S., Delgado, I., Calvo-Henriquez, C., Lavigne, P., Falanga, C., Barillari, M.,Cammaroto, G., Khalife, M., Leich, P., Souchay, C., Rossi, C., Journe, F., Hsieh, J., Edjlali, M, Carlier R., Ris, L., Lovato, A., Filippis, C., Coppee, F., Fakhry, N., Ayad, T. \& Saussez, S. (2020). Gustatory dysfunctions in COVID19. European Archives Of Oto-Rhino-Laryngology, 277(8), 2397-2398. http://dx.doi.org/10.1007/s00405-020-06154-w.

Liu, Y., Kuo, R. \& Shih, S. (2020). COVID-19: the first documented coronavirus pandemic in history. Biomedical Journal, 43(4), 328-333. http://dx.doi.org/10.1016/j.bj.2020.04.007.

Passareli, P., Lopez, M., Bonaviri, G., Garcia-Godoy, F. \& D'Addona, A. (2020). Taste and smell as chemosensory dysfunctions in COVID-19 infection. American Journal Of Dentistry, 1(1), 135-137. 
Research, Society and Development, v. 10, n. 16, e555101624107, 2021

(CC BY 4.0) | ISSN 2525-3409 | DOI: http://dx.doi.org/10.33448/rsd-v10i16.24107

Pedrosa, M., Sipert, C. \& Nogueira, F. (2020). Altered taste in patients with COVID-19: the potential role of salivary glands. Oral Diseases, 27(3), 798-800. http://dx.doi.org/10.1111/odi.13496.

Recalcati, S. (2020). Cutaneous manifestations in COVID-19: a first perspective. Journal Of The European Academy Of Dermatology And Venereology, 34(5), 212-213. http://dx.doi.org/10.1111/jdv.16387.

Riad, A., Kassem, I., Hockova, B., Badrah, M. \& Klugar, M. (2020). Tongue ulcers associated with SARS-CoV-2 infection: a case series. Oral Diseases, 27(3), 001-3. http://dx.doi.org/10.1111/odi.13635.

Santos, J., Normando, A., Silva, R., Paula, R., Cembranel, A., Santos-Silva, A. \& Guerra, E. (2020). Oral Manifestations in Patients with COVID-19: a living systematic review. Journal Of Dental Research, 100(2), 141-154. http://dx.doi.org/10.1177/0022034520957289.

Santos, J., Normando, A., Silva, R., Acevedo, A., Canto, G., Sugaya, N., Santos-Silva, A.R., \& Guerra, E. (2020). Oral mucosal lesions in a COVID-19 patient: new signs or secondary manifestations? International Journal Of Infectious Diseases, 97(1), 326-328. http://dx.doi.org/10.1016/j.ijid.2020.06.012.

Santosh, A. \& Muddana, K. (2020). Viral infections of oral cavity. Journal of Family Medicine And Primary Care, 9(1), 36. http://dx.doi.org/10.4103/jfmpc.jfmpc_807_19.

Sinadinos, A. \& Shelswell, J. (2020). Oral ulceration and blistering in patients with COVID-19. Evidence-Based Dentistry, 21(2), 49-49. http://dx.doi.org/10.1038/s41432-020-0100-z.

Sinjari, B., D’Ardes, D., Santilli, M., Rexhepi, I., D’Addazio, G., Carlo, P., Chiacchiaretta, P., Caputi, S. \& Cipollone, F. (2020). SARS-CoV-2 and Oral Manifestation: an observational, human study. Journal Of Clinical Medicine, 9(10), 3218. http://dx.doi.org/10.3390/jcm9103218. 